\section{Dissolution Enhancement of Ibuprofen Solid Dispersion Prepared with Vinyl Polymers by Fusion Method}

\author{
*Naz Hasan Huda ${ }^{1}$, Nadia Saffoon ${ }^{2}$ and Yeakuty Marzan Jhanker ${ }^{2}$ \\ ${ }^{1}$ Department of Pharmacy, Stamford University Bangladesh \\ 51, Siddeswari Road, Dhaka-1217, Bangladesh. \\ ${ }^{2}$ Department of Pharmacy, The University of Asia Pacific \\ Dhaka-1209, Bangladesh.
}

\author{
*Corresponding Author \\ Naz Hasan Huda \\ Assistant Professor \\ Department of Pharmacy \\ Stamford University Bangladesh \\ 51, Siddeswari Road \\ Dhaka-1217, Bangladesh. \\ Contact no.: +8801199027212 \\ E-mail: nazhasan@gmail.com
}

\begin{abstract}
Solid dispersions of ibuprofen are prepared by the fusion method aiming to enhance its dissolution properties. Three vinyl polymers named Kollidone VA64, Povidone K12 and Povidone K30, are used in this work. In vitro dissolution studies were carried out to evaluate the dispersions. The outcome of the study indicated that the rate of dissolution of ibuprofen was considerably improved when formulated in solid dispersions with Povidone K12 and Povidone K30. Solid dispersions with Kollidone VA64 showed drug retarding capability and can be used to formulate sustained release dosage form.
\end{abstract}

Key words: Ibuprofen, Dissolution rate, Solid dispersion, Fusion method, Kollidone, Povidone.

\title{
INTRODUCTION
}

Fast onset of action is often desirable for ibuprofen, a widely used non-steroidal anti-inflammatory drug (NSAID) to be utilized in the treatment of mild to moderate pain and fever (Rainsford, 2003). The drug's rapid pharmacologic action can be obtained by tweaking its formulation so that the plasma drug concentration reaches optimum level within a short period of time (Buxton, 2006). Newa et al. stated that for existing dosage forms of ibuprofen, absorption of the drug is controlled primarily by its dissolution rate and because its high membrane permeability characteristic, extent of ibuprofen absorption approaches up to $100 \%$ upon dissolution.

Enhancing solubility and dissolution rate of poorly water-soluble drugs like ibuprofen is one of the striking areas of research in pharmaceutical field (Dhirendra et al., 2009). Various strategies have been utilized including prodrug formation (Murtha and Ando, 1994), complexation (Ghorab and Adeyeye, 2001), microcapsulation (Adeyeye et al., 1994), the use of surfactants, lipids, permeation enhancers, micronization, salt formation, cyclodextrines, nanoparticles, solid dispersions, self emulsifying drug delivery system (Shakhtshneider et al., 1996; Craig, 2002; Gao and Morozowich, 2006; Rane et al., 2007; Tang et al., 2007) etc. to overcome the problems associated with oral absorption and bioavailability issue. However ahead of all, solid dispersion is the most promising method to the scientists due to the ease of preparation, ease of optimization and reproducibility of the manufacturing method (Chiou and Reigelman, 1971; Ford, 1986; Law et al., 1991; Leuner and Dressman, 2000). Many methods like spray drying, co-precipitation, co-evaporation and freeze dying are used for solid dispersion manufacturing; however, costly equipments are required along with complicated procedures. Apart from these techniques, fusion method is a method of choice because it is environmentally friendly, cost effective, represents no stability nor toxicity problems and can be easily scaled up for commercial purpose (Uddin et al., 2010; Bhandari et al., 2007).

The aim of this study was to prepare different solid dispersions of ibuprofen by fusion method with three vinyl polymers; Kollidone VA64, Povidone K12 and Povidone K30 and characterize them by dissolution studies to evaluate the effect of these carriers on solubility profile of ibuprofen. 


\section{MATERIALS AND METHODS}

Experimental material: Ibuprofen RS was gift sample from Orobinder, India. Kollidone VA64, Povidone K12 and Povidone K30 were obtained from BASF, Germany. Reagent: Di-sodium hydrogen phosphate and Sodium hydroxide (Merck, Germany); all other ingredients used were of analytical grade. Equipment: USP dissolution tester-Apparatus-II (VEEGO, India); UV Spectrophometer (UV mini-1204, SHIMADZU CORP., Koyoto, Japan); Digital pH meter (pH 211 Microprocessor $\mathrm{pH}$ Meter, HANNA Instruments, Romania); Electronic balance (AY 120, SHIMADZU CORP., Koyoto, Japan); Sieve (Endecott's Test Sieve, Endecotts Limited, England); glass vials, water bath, dessicator etc.

\section{Preparation of the solid dispersion}

Fusion method was used for the preparation of solid dispersions of ibuprofen (Dhirendra et al., 2009; Vasconcelos et al., 2007). Required amount of drug and polymer (Table 1) were mixed in glass vials. The mixture was then heated till it was completely melted. The temperature was maintained to a range of $80^{\circ} \mathrm{C}-90^{\circ} \mathrm{C}$. Continuous stirring during the melting was carried out to prevent the separation of the constituents. The melt was then rapidly solidified. The formulations were kept in a dessicator for further treatment. The solidified mass was then crushed, size reduced in a mortar and pestle and sieved through a 150 micron sieve. All glass vials were labeled with care and kept in dessicator. Samples for dissolution studies were taken from the vials.

Table 1: Different ratios of ibuprofen with Kollidone VA64, Povidone K12 and Povidone K30.

\begin{tabular}{|c|c|c|c|c|c|c|c|c|c|c|c|c|c|c|c|}
\hline & $\underset{\nwarrow}{\Xi}$ & $\underset{⿱}{\mathbb{Z}}$ & $\underset{⿱}{\infty}$ & $\underset{\Xi}{\Xi}$ & $\underset{\Downarrow}{\mathbb{1}}$ & $\frac{5}{N}$ & $\underset{\mathbf{N}}{\mathbf{N}}$ & $\stackrel{m}{\underset{n}{\infty}}$ & $\begin{array}{l}+ \\
N \\
\frac{N}{2}\end{array}$ & $\sum_{\substack{n \\
N}}^{n}$ & 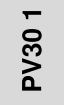 & $\begin{array}{l}N \\
\text { O } \\
\text { n }\end{array}$ & 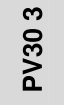 & $\begin{array}{l}+ \\
\stackrel{\text { }}{8} \\
0\end{array}$ & \begin{tabular}{l} 
10 \\
ᄋ̊ \\
\} \\
\hline $\begin{array}{l}\text { Ibuprofen } \\
\text { (mg) }\end{array}$ & 500 & 500 & 500 & 500 & 500 & 500 & 500 & 500 & 500 & 500 & 500 & 500 & 500 & 500 & 500 \\
\hline $\begin{array}{l}\text { Kollidone } \\
\text { VA64 (mg) }\end{array}$ & 125 & 250 & 500 & 1000 & 2000 & - & - & - & - & - & - & - & - & - & - \\
\hline $\begin{array}{l}\text { Povidone } \\
\text { K12 (mg) }\end{array}$ & - & - & - & - & - & 125 & 250 & 500 & 1000 & 2000 & - & - & - & - & - \\
\hline $\begin{array}{l}\text { Povidone } \\
\text { K30 (mg) }\end{array}$ & - & - & - & - & - & - & - & - & - & - & 125 & 250 & 500 & 1000 & 2000 \\
\hline
\end{tabular}
\end{tabular}

\section{Preparation of phosphate buffer $\mathrm{pH} 7.2$}

$7.34 \mathrm{gm}$ di-sodium hydrogen phosphate and $1 \mathrm{gm}$ sodium hydroxide were weighed out and dissolved in small amount of distilled water, volume was adjusted to 1 liter with the same solvent to prepare 1 liter phosphate buffer. The $\mathrm{pH}$ of the buffer solution was adjusted using a $\mathrm{pH}$ meter.

\section{In vitro dissolution Study}

These studies were conducted at $37 \pm 0.5^{\circ} \mathrm{C}$ on an USP specification dissolution rate test type II apparatus (Paddle apparatus) with six sections assembly according to the USP 30 procedure (USP 30 and NF 25, 2007). For in vitro dissolution studies, phosphate buffer $\mathrm{pH} 7.2$ was used as dissolution media. Water-bath temperature was fixed \& confirmed to be $37 \pm 0.5^{\circ} \mathrm{C}$ before starting the experiment. The medium was preheated to $37^{\circ} \mathrm{C}$ and then a quantity of $900 \mathrm{ml}$ was added to each vessel. The apparatus was then assembled and paddle rotation was started and adjusted at $100 \mathrm{rpm}$ and the system was allowed to equilibrate for 15 minutes.

After that the paddle rotation was stopped and fixed amounts of solid dispersion containing $50 \mathrm{mg}$ equivalent ibuprofen from each batch were placed in the vessels. The apparatus was immediately operated at 100rpm. Each vessel, vessel position and corresponding sample result were assigned the same code. The duration of the experiment was 60 minutes for each set of sample.

$10 \mathrm{ml}$ of sample was withdrawn from the media at pre-determined intervals of $5,10,15,20,30,45$, 60 minutes. Each and every time $10 \mathrm{ml}$ of dissolution sample was compensated by adding $10 \mathrm{ml}$ fresh phosphate buffer. The sample solutions were diluted and analyzed at $221 \mathrm{~nm}$ for ibuprofen by 
UV spectrophotometer. The amount of drug present in the samples was calculated from calibration curve constructed from the standard solution of USP reference standard test drug (Figure 1).

\section{RESULTS AND DISCUSSION}

Effects of Kollidone VA64, Povidone K12 and Povidone K30 on dissolution rate of ibuprofen solid dispersions are shown in figure 2, 3 and 4 respectively.

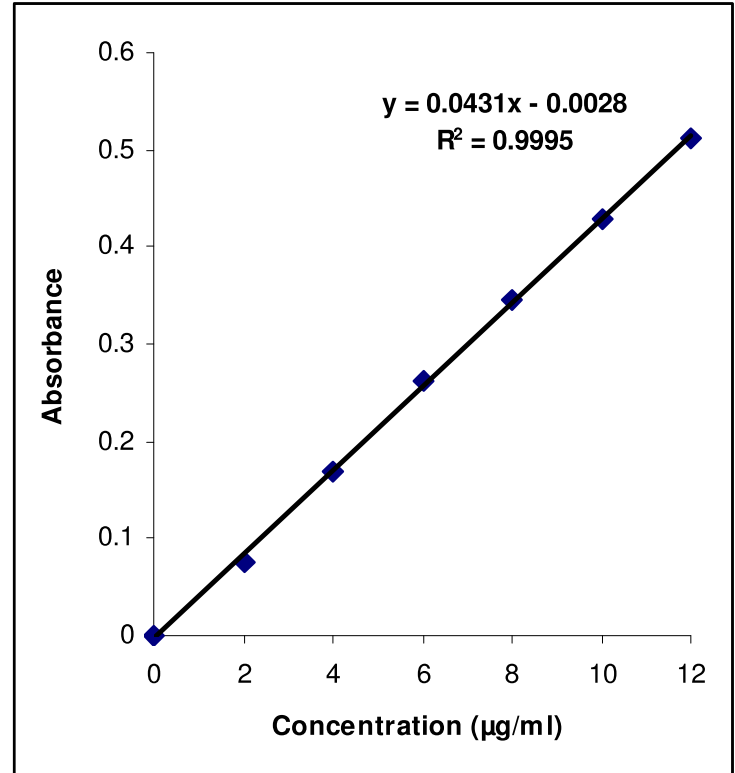

Figure 1: Calibration curve of ibuprofen using phosphate buffer pH 7.2 as media.

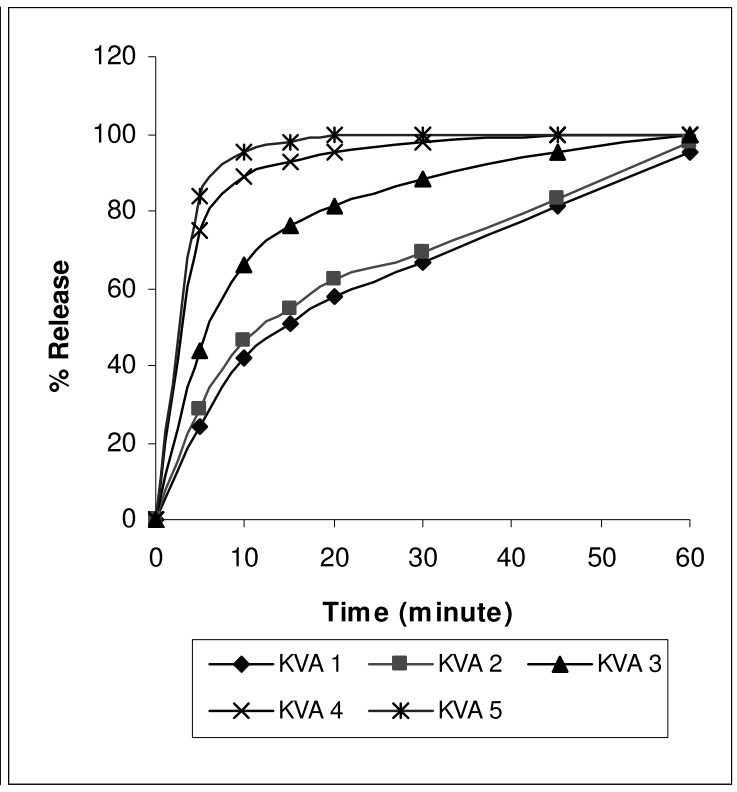

Figure 2: Percent release of ibuprofen from solid dispersions prepared in different polymer ratios of Kollidone VA64.

$100 \%$ drug release from formulations prepared with KVA 64 was obtained within 15 minutes, 30 minutes and 60 minutes when ibuprofen to polymer ratio was 1:4, 1:2 and 1:1, respectively. However, formulations prepared with 2:1 and 4:1 drug to polymer ratio did not give $100 \%$ release of drug within 60 minutes dissolution time; $97.73 \%$ and $95.52 \%$ release of ibuprofen was obtained within 60 minute respectively (Figure 2).

Povidone $\mathrm{K} 12$ based ibuprofen solid dispersions released ibuprofen within 15 minutes, 30 minutes and 45 minutes when drug to polymer ratio was 1:4, 1:2 and 1:1 respectively. Both 2:1 and 4:1 ratio formulations took 60 minutes for $100 \%$ release of the drug (Figure 3).

Solid dispersions prepared with povidone $\mathrm{K} 30$ as polymer released $100 \%$ drug within 15 minutes for drug to polymer ratios of both $1: 4$ and 1:2. It also released ibuprofen by $100 \%$ within 30 minutes, 45 minutes and 60 minutes with drug to polymer ratio of 1:1, 2:1 and $4: 1$ respectively (Figure 4).

At lower concentration of KVA 64 (Ibuprofen:KVA 64=4:1) 100\% release of ibuprofen was not obtained from KVA 64 based solid dispersions within 60 minutes (95.52\%). Even 2:1 drug to KVA 64 ratio formulation was unable to give $100 \%$ drug release within 60 minutes dissolution time (97.73\% release in 60 minutes). But when the drug to KVA 64 ratio was $1: 4,100 \%$ release of ibuprofen was obtained within 15 minutes. Povidone K30 gave a better dissolution profile in comparison to povidone K12. Povidone K30 based solid dispersions with the drug to polymer ratio of $1: 2$ and $1: 4$ released $100 \%$ ibuprofen within 60 minutes. Povidone K30 showed highest dissolution rate at $32 \%$ polymer concentration, povidone $\mathrm{K} 12$ at $50 \%$ polymer concentration and KVA64 at $65 \%$. When \%polymer was $65 \%$ all three polymers showed nearly same dissolution rate. 


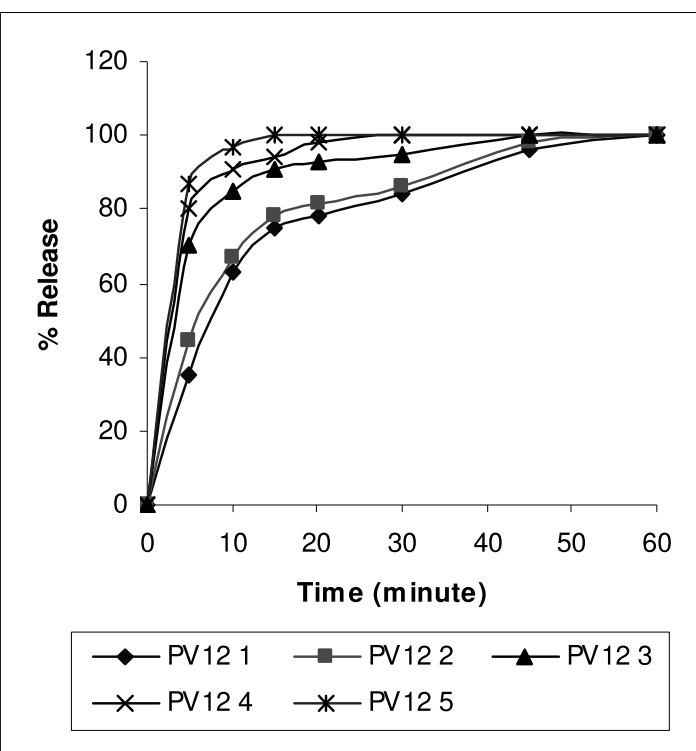

Figure 3: Percent release of ibuprofen from solid dispersions prepared in different polymer ratios of Povidone K12.

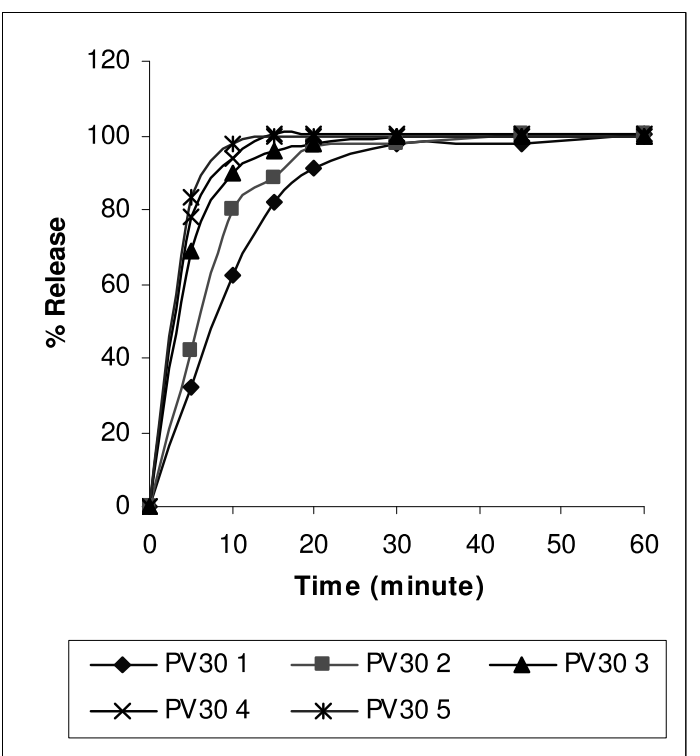

Figure 4: Percent release of ibuprofen from solid dispersions prepared in different polymer ratios of Povidone K30.

\section{CONCLUSION}

With the invention of many poorly water soluble drugs, enhanced dissolution rate i.e. improved oral bioavailability of oral solid dosage forms is a burning question these days. In our study we used fusion method to prepare solid dispersions of ibuprofen to increase their dissolution rate. Vinyl polymers (Kollidone VA64, Povidone K12 and Povidone K30) were used in the work. It is clear from the data obtained that a higher polymer concentration gave faster drug release for all the polymers used to prepare the solid dispersions. Again, for immediate release drug delivery systems, dissolution profiles obtained for Povidone K12 and Povidone K30 based solid dispersions are most encouraging because both they are capable of releasing cent percent of drug within a short period of time as discussed above. On the other hand, few of the Kollidone VA64 based preparations can be used to retard the drug release to some extent to develop time retarded drug delivery system.

\section{REFERENCES}

Adeyeye CM, Price JC. (1994), Development and evaluation of sustained-release ibuprofen-wax microspheres-II. In vitro dissolution studies. Pharm. Res. 11: 575-579.

Bhandari KH, Newa M, Kim JA, Yoo BK, Woo JS, Lyoo WS, Lim HT, Choi HG, Yong CS. (2007), Preparation, characterization and evaluation of coenzyme Q10 binary solid dispersions for enhanced solubility and dissolution, Biol. Pharm. Bull. 30: 1171-1176.

Buxton ILO. (2006), In - Goodman \& Gilman's - The Pharmacological Basis of Therapeutics (2006) $11^{\text {th }}$ Edition, McGraw-Hill Medical Publishing Division, Digital Edition, Chapter 1.

Chiou WL, Reigelman S. (1971), Pharmaceutical applications of solid dispersion systems. J Pharm Sci. 60(9): 1281-1302.

Craig DQM. (2002), The mechanisms of drug release from solid dispersions in water-soluble polymers. Int. J. Pharm. 231: 131-144.

Dhirendra K, Lewis S, Udupa N, Atin K. (2009), Solid Dispersions: A Review. Pak. J. Pharm. Sci. 22(2): 234-246.

Ford JL. (1986), The current status of solid dispersions. Pharm Acta Helv. 61:69-88. 
Gao P, Morozowich W. (2006), Development of supersaturatable self-emulsifying drug delivery system formulations for improving the oral absorption of poorly soluble drugs. Expert Opin. Drug Deliv. 3(1): 97-110.

Ghorab MK, Adeyeye MC. (2001), Enhancement of ibuprofen, dissolution via wet granulation with beta-cyclodextrin. Pharm. Dev. Technol. 6: 305-314.

Law SL, Lo WY, Lin FM, Chaing CH. (1992), Dissolution and absorption of nifedipine in polyethylene glycol solid dispersion containing phosphatidylcholine. Int. J. Pharm. 84: 161166.

Leuner C, Dressman J. (2000), Improving drug solubility for oral delivery using solid dispersions. Eur J Pharm Biopharm. 50:47-60.

Murtha JL, Ando HY. (1994), Synthesis of the cholesteryl ester prodrugs cholesteryl ibuprofen and cholesteryl flufenamate and their formulation into phospholipid microemulsions. J. Pharm. Sci. 83: 1222-1228.

Newa M, Bhandari KH, Kim JA, Yoo BK, Choi HG, Yong CS, Woo JS, Lyoo SK. (2008), Preparation and evaluation of fast dissolving ibuprofen-polyethylene glycol 6000 solid dispersions. Drug Delivery. 15:355-364.

Rainsford KD. (2003), Discovery, mechanisms of action and safety of ibuprofen. Int J Clin. Pract. Suppl. 135: 3-8.

Rane Y, Mashru R, Sankalia M, Sankalia J. (2007), Effect of hydrophilic swellable polymers on dissolution enhancement of carbamazepine solid dispersions studied using response surface methodology. AAPSPharmSciTech. 8(2): Article 27.

Shakhtshneider TP, Vasiltchenko MA, Politov AA, Boldyrev VV. (1996), The mechanochemical preparation of solid disperse systems of ibuprofen-polyethylene glycol. Int. J. Pharm. 130: 25-32.

Tang J, Sun J, He ZG. (2007), Self-emulsifying drug delivery systems: strategy for improving oral delivery of poorly soluble drugs. Current Drug Therapy. 2: 85-93.

Uddin R, Saffoon N, Huda NH, Jhanker YM. (2010), Effect of water soluble polymers on dissolution enhancement of ibuprofen solid dispersion prepared by fusion method. $S \mathrm{~J}$ Pharm Sci. 3(1): 63-67.

United States Pharmacopeia 30 and National Formulary 25. (2007), The United States Pharmacopeial Convention, CD ROM.

Vasconcelos T, Sarmento B, Costa P. (2007), Solid dispersions as strategy to improve oral bioavailability of poor water soluble drugs. Drug Discovery Today. 12(23-24): 1068-1075. 